\title{
Growth trajectories and asthma/rhinitis in children: a longitudinal study in Taiwan
}

\author{
Yang-Ching Chen ${ }^{1,2,7}$, Tsan-Hon Liou ${ }^{3}$, Pau-Chung Chen ${ }^{4,5}$, Bor-Luen Chiang ${ }^{6}$, \\ Yao-Hsu Yang ${ }^{6}$, Hsien-Yu Fan ${ }^{1}$ and Yungling Leo Lee ${ }^{7,8}$
}

Affiliations: ${ }^{1}$ Taipei City Hospital, ZhongXing Branch, Taipei, Taiwan. ${ }^{2}$ School of Nutrition and Health Sciences, College of Nutrition, Taipei Medical University, Taipei, Taiwan. ${ }^{3}$ Graduate Institute of Injury Prevention and Control, Taipei Medical University, Taipei, Taiwan. ${ }^{4}$ Institute of Occupational Medicine and Industrial Hygiene and Dept of Public Health, National Taiwan University College of Public Health, Taipei, Taiwan. ${ }^{5}$ Dept of Environmental and Occupational Medicine, National Taiwan University Hospital and National Taiwan University College of Medicine, Taipei, Taiwan. ${ }^{6}$ Dept of Pediatrics, National Taiwan University Hospital, Taipei, Taiwan. ${ }^{7}$ Institute of Epidemiology and Preventive Medicine, National Taiwan University College of Public Health, Taipei, Taiwan. ${ }^{8}$ Institute of Biomedical Sciences, Academia Sinica, Taipei, Taiwan.

Correspondence: Yungling Leo Lee, 17 Xuzhou Road, Zhongzheng District, Institute of Epidemiology and Preventive Medicine, College of Public Health, National Taiwan University, Taipei 100, Taiwan.

E-mail: leoleedntu.edu.tw

@ERSpublications

Persistently overweight children had a long-term risk of incident asthma and rhinitis in late adolescence http://ow.ly/Ixay3049PYL

Cite this article as: Chen Y-C, Liou T-H, Chen P-C, et al. Growth trajectories and asthma/rhinitis in children: a longitudinal study in Taiwan. Eur Respir J 2017; 49: 1600741 [https://doi.org/10.1183/ 13993003.00741-2016].

ABSTRACT Studies have reported the effect of body weight in early childhood on asthma. However, the effect of growth patterns during school age on asthma and rhinitis has yet to be explored. We sought to investigate whether various growth patterns predict incident asthma and rhinitis.

We conducted a nationwide longitudinal study (Taiwan Children Health Study) in 14 Taiwanese communities. Body mass index (BMI) z-scores of 4422 children aged 6-11 years were collected annually and distinct growth trajectory classes were identified using a latent generalised mixture model. Pulmonary function and exhaled nitric oxide fraction $(\mathrm{FeNO})$ levels were also measured. Whether different growth trajectory classes predict incident asthma and rhinitis at age 12, 15 and 18 years was determined using a discrete time hazard model.

Four growth trajectory classes were identified. Persistently overweight children exhibited significantly increased risks of asthma and rhinitis at age 12 years. Furthermore, being persistently overweight had a long-term effect on incident asthma (hazard ratio 2.47, 95\% CI 1.18-5.12) and rhinitis (hazard ratio 1.44, 95\% CI 1.12-1.84) in adolescence and early adulthood. Children in high BMI classes exhibited significantly lower pulmonary functions compared with normal growth children. FeNO levels were lower in children in the high BMI classes and higher in children showing declining obesity compared with normal growth children.

Persistently overweight children exhibited incident asthma and rhinitis in adolescence and early adulthood.

This article has supplementary material available from erj.ersjournals.com

Received: April 132016 | Accepted after revision: Aug 312016

Support statement: This study was supported by grants (101-2314-B-532-002-MY3 and 104-2314-B-532-002-MY3) from the Ministry of Science and Technology in Taiwan.

Conflict of interest: None declared.

Copyright OERS 2017 


\section{Introduction}

Growing evidence supports the association between obesity and asthma in children [1]. In addition, most prospective studies have suggested that obesity antedates the development of asthma [2]. However, growth is dynamic in children. Using body mass index (BMI) trajectories can better describe the longitudinal development of a population, as well as the variation of individual development, compared with a single measurement of BMI. In addition, previous studies using concurrent BMI as a predictor for asthma might have problems in reverse causality [3]. BMI developmental course might better reflect the time period of gaining excess weight and the cumulative risk of asthma development. Considering the current obesity pandemic [4], determining whether elevated BMI in childhood, adolescence or young adulthood contributes independently to the risk of asthma is crucial.

Researchers have explored the associations between growth trajectory and asthma, but such studies were conducted on populations with different age ranges by using different analytical methodologies. A birth cohort study following children from 1 to 18 years of age reported that children with early persistent obesity since age 1-4 years exhibited a 2.2-fold increased risk of asthma compared with those with normal weight [5]. Furthermore, the critical period for a different growth pattern was age 1-4 years. A European study conducted on a large joint birth cohort [6] reported that children with a rapid BMI gain trajectory class in the first 2 years of life exhibited a higher risk of incident asthma within the first 6 years of life than did children with a less pronounced weight gain slope in childhood. Previous studies have focused on adiposity measurements during infancy and early childhood. However, no study has explored the relationships between growth trajectories during school age and incident asthma. The history of asthma during adolescence is different from that during early childhood [7].

Allergic rhinitis is one of the most common atopic diseases among children and its prevalence is increasing worldwide [8]. 80\% patients with allergic rhinitis exhibit symptoms of rhinitis since childhood and rhinitis mainly affects children aged 9-17 years [9]. Allergic rhinitis can severely affect the quality of life of children and their performance in school [10]. However, in contrast to reports on the strong association between obesity and asthma [11], studies on obesity and allergic rhinitis have reported inconsistent results $[12,13]$. No study has investigated the effect of growth trajectories on allergic rhinitis. Adiposity-related increased level of inflammation and oxidative stress in airways of children might induce the occurrence of allergic rhinitis [14].

We performed a nationwide longitudinal study (Taiwan Children Health Study (TCHS)) in 14 representative communities in Taiwan. To investigate which growth pattern during school age may be associated with the risk of asthma and allergic rhinitis, we examined the growth trajectories at age 6-11 years and their relation to active asthma and allergic rhinitis at age 12 years. We then used these growth classes in the second step as a long-term predictor for incident asthma and allergic rhinitis at age 12, 15 and 18 years in a survival model. Furthermore, the TCHS data can be used to analyse the differences in pulmonary function performance levels and airway inflammation status among various growth trajectory classes.

\section{Methods}

\section{Study design and data collection}

We conducted the nationwide TCHS on children from elementary and middle schools in 14 Taiwanese communities during 2007-2010. The TCHS involved a multipurpose nationwide design comprising two cohorts, and it focused on atopic diseases, adiposity growth, pubertal development, and certain social and behavioural issues in children [15]. Cohort 1 enrolled 12-year-old children and Cohort 2 enrolled 10-year-old children. The baseline response rates for Cohorts 1 and 2 were $86.5 \%$ and $75.5 \%$, respectively. In Cohort 1, follow-up surveys were conducted on children aged 15 and 18 years. In Cohort 2, follow-up surveys were conducted on children aged 11 and 12 years. The parents or guardians of each participating student provided written informed consent and completed a written questionnaire. The TCHS protocol was approved by the Institutional Review Board at National Taiwan University Hospital and it complied with the principles outlined in the Declaration of Helsinki [16].

\section{BMI measurements}

In both cohorts, the growth trajectory was assessed annually at age 6-11 years by using BMI $\left(\mathrm{kg} \cdot \mathrm{m}^{-2}\right.$; $\mathrm{n}=4422$ ). From 2010 to 2012, the BMI levels of Cohort 2 were measured annually during school visits [17]. For earlier data on BMI growth in both cohorts, body height and weight data were retrospectively retrieved from the participants' elementary school health records. The BMI data were transformed into standardised BMI z-scores according to the World Health Organization (WHO) growth standards for school-aged children and adolescents $[18,19]$. The prevalences of overweight and obesity were defined according to three criteria: Taiwanese growth charts [20], WHO [19] and International Obesity Task Force 
(IOTF) criteria [21]. A child being overweight was defined by BMI between the 85th and 95th percentile of age- and sex-specific BMI, and childhood obesity was defined by BMI $\geqslant 95$ th percentile of age- and sex-specific BMI according to the growth charts for children.

\section{Outcome definition}

A parent-reported questionnaire was used for defining phenotypes of asthma and rhinitis in children aged 12 and 15 years [22]. Active asthma cases were distinguished from normal children by asking two questions: "Has a doctor ever diagnosed your child as having asthma?" and "Did your child ever experience difficulty breathing, or did you observe any wheezing or whistling from his or her chest in the past 12 months?". If the answers were "Yes" to both questions, we classified the child as an active asthma case. Moreover, exercise-induced asthma was defined as a positive answer to the question "Did your child ever experience whistling from his or her chest during or after exercise in the past 12 months?". Allergic rhinitis was also determined by a positive answer to the question "Has a doctor ever diagnosed your child as having allergic rhinitis?". If the answer was "Yes", we then asked when was the first time that the child had been diagnosed with allergic rhinitis. We clarified active allergic rhinitis cases as diagnosed in the past 1 year. We obtained the answers to the same questions from children aged 18 years in the survey, rather than using a parent-reported questionnaire. We initially combined Cohorts 1 and 2 to determine which growth trajectory class across the ages 6-11 years would affect active asthma/rhinitis at age 12 years. We followed up Cohort 2 children until age 12 years; therefore, to determine whether various growth trajectory classes across the ages 6-11 years would predict incident asthma/rhinitis phenotypes in later life (at age 12, 15 and 18 years), we can only use Cohort 1.

In the questionnaire, we also identified the age of initial physician-diagnosed asthma/rhinitis. For the participants in Cohort 1, incident asthma phenotypes were calculated by excluding the history of asthma before age 11 years $(n=113)$. Incident allergic rhinitis was calculated by excluding the history of rhinitis before age 11 years $(n=204)$. Incident asthma/rhinitis phenotypes were determined when the answer was "Yes" to ever physician-diagnosed asthma/rhinitis and exercise-induced asthma questions during the follow-up period.

We used exhaled nitric oxide fraction ( $F$ eNO) levels as a surrogate marker of airway inflammation [23]. One NIOX MINO Airway Inflammation Monitor (Aerocrine, Solna, Sweden) was used in the 2012 survey of Cohort 2. The standard procedures for measuring the FeNO levels were based on the recommendations made by the American Thoracic Society and European Respiratory Society [24].

Pulmonary function tests were conducted according to our previously standardised protocol [25]. The forced expiratory volume in $1 \mathrm{~s}$ (FEV1)/forced vital capacity (FVC) ratio demonstrated the most significant associations with BMI [17]. Therefore, the FEV1/FVC ratio was used as a surrogate marker for pulmonary function performance.

\section{Statistical analysis}

In the TCHS, we identified distinct growth trajectory patterns across the ages 6-11 years according to individual BMI z-score trajectories by using the latent growth mixture model (LGMM) [26]. The LGMM, an extension of random effects of the latent class growth model, refers to modelling with latent variables that are used to identify heterogeneous subgroups with similar developmental trajectories (online supplementary figure E1) [27]. The random effects are allowed to have different distributions among individuals belonging to different trajectory classes. Missing data were appropriately handled in the LGMM with the assumptions of missing at random. Furthermore, to assess model adequacy, the Bayesian Information Criterion (BIC) and Lo-Mendell-Rubin (LMR) tests were used [28]. Lower BIC test values, with significant results in the LMR test for a $k$-class model compared with a $k$-1-class model, suggested that the model fitted the data adequately when an additional latent class was included. LGMM analysis was conducted using Mplus software version 7 (www.statmodel.com).

For the present study, we attempted initially to determine which growth trajectory class across the ages 6-11 years would affect active asthma/rhinitis at age 12 years in the TCHS by using multivariate logistic regression. Our statistical models were adjusted for several potential confounders, such as sex, age, parental education, family income, household environmental tobacco smoke, birthweight, gestational age, breastfeeding and asthma oral steroid use. Moreover, to determine whether various growth trajectory classes across the ages 6-11 years would predict incident asthma/rhinitis phenotypes in later life (at age 12, 15 and 18 years), we used the discrete time hazard model (DTHM) [29] in Cohort 1. The DTHM is a survival analysis model that enables estimating hazard ratios (HRs) and 95\% confidence intervals when an event occurrence may be considered in a discrete period. The model was weighted by individual class probabilities for each of the four trajectory classes to account for the uncertainty in classifying a child into a particular trajectory class. The comparisons of FEV1/FVC ratios and FeNO levels among different growth 
trajectory classes were analysed using multivariate ANOVA. Descriptive analysis, logistic regression and DTHM analysis were conducted using SAS software version 9.3 (www.sas.com). On the basis of a two-sided estimation, statistical significance was set at 5\%.

\section{Results}

In this study, the TCHS population comprised 4422 children with available data for both growth trajectory classes (age 6-11 years) and asthma/rhinitis phenotypes (age 12 years). Table 1 presents the characteristics of participants aged 12 years. Although Cohorts 1 and 2 were enrolled from the same 14 communities, the participants aged 12 years were recruited in 2007 and 2012, respectively. During this 5-year time gap, we observed a higher level of parental education and family income in Cohort 2 as compared with Cohort 1. In the TCHS, $4.0 \%$ of children aged 12 years had active asthma, 5.9\% had exercise-induced asthma and 9.7\% had active allergic rhinitis. In Cohort 1 , the follow-up rate was $93.5 \%$ for children aged 15 years and 71.3\% for children aged 18 years. When children with a history of asthma were excluded, the incidences of asthma were 10.6, 4.7 and 6.6 per 1000 person-years at age 12, 15 and 18 years, respectively. When children with a history of allergic rhinitis were excluded, the incidences of physician-diagnosed allergic rhinitis were $62.1,20.4$ and 21.4 per 1000 person-years at age 12,15 and 18 years, respectively.

\section{Growth trajectory classes}

After conducting model adequacy assessment through the BIC and LMR tests [28], we determined four growth trajectory classes (figure 1). Both cohorts demonstrated similar trajectory patterns (online supplementary figures E2 and E3); therefore, these cohorts were combined to further analyse the growth trajectories at age 6-11 years and their relations to active asthma and allergic rhinitis at age 12 years. Online supplementary tables E1 and E2 list the detailed estimates of growth parameters in the four trajectory classes derived through the LGMM, and online supplementary table E3 lists the model fit indices. The first trajectory class (Class 1, $\mathrm{n}=2098,47.4 \%$ ) was characterised by children with normal growth, aged 6-11 years and demonstrating BMI z-scores of nearly zero. However, when three criteria were used to define overweight or obese children (table 2), 5.6\% of the children in Class 1 were identified

\section{TABLE 1 Characteristics of participants aged 12 years in the Taiwan Children Health Study}

\begin{tabular}{|c|c|c|c|}
\hline & Cohort 1 & Cohort 2 & Cohorts 1 and 2 \\
\hline Subjects & 1008 & 3414 & 4422 \\
\hline Age years & $12.7 \pm 0.3$ & $12.1 \pm 0.3$ & $12.3 \pm 0.4$ \\
\hline Male & 503 (49.9) & $1736(50.9)$ & $2239(50.6)$ \\
\hline BMI $\mathrm{kg} \cdot \mathrm{m}^{-2}$ & $20.6 \pm 4.3$ & $19.0 \pm 3.9$ & $19.4 \pm 4.0$ \\
\hline \multicolumn{4}{|l|}{ Parental education years } \\
\hline Senior high school or below & 668 (66.3) & 1785 (52.3) & $2453(55.5)$ \\
\hline College or university & 305 (30.3) & $1344(39.4)$ & 1649 (37.3) \\
\hline Post-graduate school & $35(3.5)$ & $285(8.4)$ & $320(7.2)$ \\
\hline \multicolumn{4}{|l|}{ Family income NTD } \\
\hline$<600000$ & $614(60.9)$ & $1657(48.5)$ & $2271(51.4)$ \\
\hline $600001-1000000$ & $277(27.5)$ & $1175(34.4)$ & 1452 (32.8) \\
\hline$\geqslant 1000001$ & $117(11.6)$ & $582(17.1)$ & $699(15.8)$ \\
\hline Birthweight $\mathbf{g}$ & $3193.2 \pm 445.5$ & $3132.2 \pm 455.8$ & $3150.4 \pm 453.5$ \\
\hline Gestational age weeks & $39.1 \pm 1.4$ & $38.5 \pm 1.8$ & $38.7 \pm 1.7$ \\
\hline Breastfeeding & $468(46.4)$ & $1213(51.8)$ & $1681(50.2)$ \\
\hline Oral steroid use in past 1 year & $11(1.1)$ & $19(0.56)$ & $30(0.68)$ \\
\hline Household cigarette smoke & $468(46.4)$ & $1252(36.7)$ & 1720 (38.9) \\
\hline Active asthma & $34(3.4)$ & $122(4.2)$ & $156(4.0)$ \\
\hline Exercise-induced asthma & $44(4.4)$ & $193(6.5)$ & $237(5.9)$ \\
\hline Active allergic rhinitis & $32(3.2)$ & 349 (11.9) & 381 (9.7) \\
\hline FEV1/FVC \% & $91.0(5.4)$ & $91.9(6.6)$ & $91.7(6.3)$ \\
\hline FenO ppb & & $11.7(18.0)$ & \\
\hline \multicolumn{4}{|l|}{ Trajectory class } \\
\hline Class 1 & 446 (44.3) & $1652(48.4)$ & $2098(47.4)$ \\
\hline Class 2 & $209(20.7)$ & $671(19.7)$ & $880(19.9)$ \\
\hline Class 3 & $322(31.9)$ & $1008(29.5)$ & $1330(30.1)$ \\
\hline Class 4 & $31(3.1)$ & $83(2.4)$ & $114(2.6)$ \\
\hline $\begin{array}{l}\text { All data are presented as } n, n \\
\text { number because of missing da } \\
\text { volume in } 1 \mathrm{~s} \text {; FVC: forced vital }\end{array}$ & $\begin{array}{l}n(\%) . \text { The } r \\
\text { dy mass inde }\end{array}$ & $\begin{array}{l}\text { participants } \\
\text { W Taiwan do } \\
\text { fraction. }\end{array}$ & $\begin{array}{l}\text { up to the total } \\
\text { orced expiratory }\end{array}$ \\
\hline
\end{tabular}




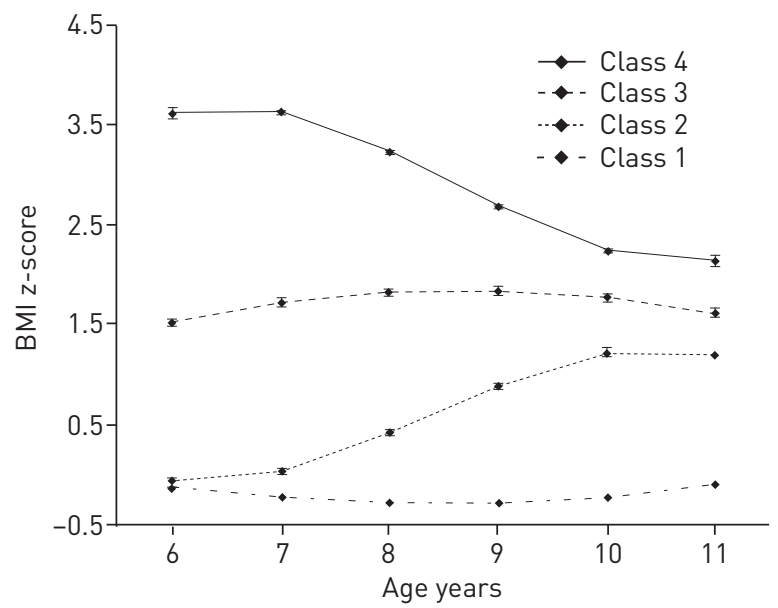

FIGURE 1 Age- and sex-specific body mass index (BMI) z-score trajectory classes across the ages 6-11 years, which were estimated using the latent growth mixture model. Class 1: normal growth (47.4\%); Class 2: rapid growth (19.9\%); Class 3: persistently overweight (30.1\%); Class 4: declining obesity $(2.6 \%)$. Bars indicate $95 \%$ confidence intervals around the estimated values in each trajectory class.

as overweight at age 11 years according to the Taiwanese criteria. An increased prevalence of overweight (20.2\%) was observed in normal growth children according to the IOTF criteria, suggesting that the IOTF criteria might overestimate the prevalence of overweight in normal growth children in Taiwan.

In our analysis, 880 rapidly growing children (19.9\%) were categorised into Class 2. At age 6 years, children in Class 2 exhibited similar BMI z-scores to those of children in Class 1 . However, because of the steepest BMI z-scores elevation, the BMI z-scores of children in Class 2 at age 11 years were $>1$.

Class 3 ( $n=1330,30.1 \%)$, involving persistently overweight children, registered BMI $\mathrm{z}$-scores indicative of long-term overweight and obesity at age 6-11 years. Children in Class 3 experienced a marginal increase in their BMI z-scores at age 6-8 years and stabilised high BMI z-scores after age 8 years.

\section{TABLE 2 Prevalence of overweight or obese status across the ages $6-11$ years by four trajectory classes ${ }^{\#}$}

\begin{tabular}{|c|c|c|c|c|c|c|c|c|c|c|c|c|}
\hline \multirow[t]{2}{*}{ Age years } & \multicolumn{3}{|c|}{ Class 1} & \multicolumn{3}{|c|}{ Class 2} & \multicolumn{3}{|c|}{ Class 3} & \multicolumn{3}{|c|}{ Class 4} \\
\hline & Taiwan & WHO & IOTF & Taiwan & WHO & IOTF & Taiwan & WHO & IOTF & Taiwan & WHO & IOTF \\
\hline \multicolumn{13}{|c|}{ Normal weight } \\
\hline 7 & 98.8 & 94.4 & 88.1 & 96.2 & 86.5 & 80.3 & 34.0 & 13.2 & 6.2 & 5.3 & 2.5 & 0.0 \\
\hline 8 & 99.8 & 96.7 & 91.0 & 86.6 & 71.7 & 63.5 & 26.9 & 5.9 & 2.0 & 9.6 & 2.2 & 2.3 \\
\hline 9 & 99.4 & 96.4 & 90.6 & 67.1 & 52.8 & 41.7 & 23.0 & 5.4 & 2.2 & 17.3 & 13.5 & 11.9 \\
\hline \multicolumn{13}{|c|}{ Overweight } \\
\hline 6 & 3.3 & 5.9 & 14.3 & 4.6 & 8.1 & 16.3 & 38.8 & 26.1 & 42.9 & 18.9 & 8.6 & 10.9 \\
\hline 7 & 1.2 & 5.1 & 11.7 & 3.3 & 10.2 & 18.1 & 40.4 & 24.3 & 40.0 & 20.2 & 5.3 & 9.8 \\
\hline 8 & 0.2 & 3.3 & 9.0 & 11.0 & 15.6 & 29.3 & 39.0 & 26.7 & 40.8 & 15.8 & 6.8 & 9.8 \\
\hline 9 & 0.6 & 3.4 & 9.3 & 22.3 & 19.1 & 39.1 & 35.1 & 24.6 & 38.6 & 13.6 & 4.7 & 10.3 \\
\hline 10 & 4.5 & 5.3 & 14.5 & 25.3 & 21.7 & 42.2 & 26.3 & 21.1 & 37.9 & 16.9 & 11.9 & 18.7 \\
\hline 8 & 0.0 & 0.0 & 0.0 & 2.4 & 12.7 & 7.2 & 34.1 & 67.4 & 57.2 & 74.6 & 91.0 & 87.9 \\
\hline 9 & 0.0 & 0.2 & 0.1 & 10.6 & 28.1 & 19.2 & 41.9 & 70.0 & 59.2 & 69.1 & 81.8 & 77.8 \\
\hline 10 & 3.9 & 7.0 & 5.2 & 16.1 & 35.0 & 25.4 & 36.3 & 54.3 & 44.4 & 55.7 & 70.3 & 64.4 \\
\hline 11 & 6.3 & 10.4 & 8.6 & 20.7 & 34.8 & 26.0 & 36.4 & 46.9 & 40.2 & 58.7 & 65.3 & 63.6 \\
\hline
\end{tabular}

Data are presented as \%. Definitions of overweight or obesity were demonstrated by three different criteria: Taiwan: new growth charts for Taiwanese children; WHO: World Health Organization Growth Standards; IOTF: International Obesity Task Force Asian cut-off point. \# : Class 1: normal growth ( $n=2098)$; Class 2: rapid growth ( $n=880)$; Class 3: persistently overweight $(n=1330)$; Class 4: declining obesity ( $n=114)$. 
TABLE 3 Odds ratios of asthma/rhinitis phenotypes according to the various growth trajectory classes in children aged 12 years (Cohorts 1 and 2)

\begin{tabular}{lccc} 
Trajectory class & Active asthma & Exercise-induced asthma\# & Active allergic rhinitis $^{\text {}}$ \\
\hline Class 1 (reference) & 1 & 1 & 1 \\
Class 2 & $1.29(0.82-2.02)$ & $1.12(0.77-1.61)$ & $1.12(0.90-1.38)$ \\
Class 3 & $1.54(1.05-2.26)$ & $1.42(1.04-1.93)$ & $1.25(1.03-1.53)$ \\
Class 4 & $0.85(0.25-2.87)$ & $0.96(0.37-2.44)$ & $1.11(0.69-1.77)$
\end{tabular}

Data are presented as odds ratios with $95 \%$ confidence intervals. See figure 1 and table 2 for details of trajectory classes. \#: logistic regression models were adjusted for sex, age, parental education, family income, household environmental tobacco smoke, birthweight, gestational age, breastfeeding and asthma oral steroid use; ": logistic regression models were adjusted for sex, age, parental ducation, family income, household environmental tobacco smoke, birthweight, gestational age and breastfeeding.

In contrast to the children in Classes 2 and 3, those in Class 4 ( $\mathrm{n}=114,2.6 \%)$ demonstrated a significant decline in the BMI z-scores from $>3.5$ to $<3$. These few children who were originally considered extremely obese might experience a natural growth in height or a lifestyle modification, thus reducing their BMI z-scores.

\section{Growth trajectory and asthma}

The children in Class 3 exhibited significantly increased risks of active asthma (odds ratio (OR) 1.54, 95\% CI 1.05-2.26) and exercise-induced asthma (OR 1.42, 95\% CI 1.04-1.93) (table 3). Table 4 details the survival analysis of the effect of the BMI trajectory across the ages 6-11 years on incident asthma during adolescence or early adulthood. After excluding children with a history of asthma, being in Class 3 (i.e. the persistently overweight trajectory class) would significantly increase the risk of incident asthma (HR 2.47, 95\% CI 1.18-5.12) and incident exercise-induced asthma (HR 1.40, 95\% CI 1.01-1.95).

\section{Growth trajectory and allergic rhinitis}

The children in Class 3 exhibited a significantly increased risk of active allergic rhinitis (OR 1.25, 95\% CI 1.03-1.53) (table 3). When these children were followed longitudinally up to age 18 years, we determined that they exhibited an increased risk of incident allergic rhinitis (HR 1.44, 95\% CI 1.12-1.84) (table 4).

\section{Pulmonary function test and airway inflammation in four growth trajectory classes}

Our data suggested an inverse relationship between the growth trajectory classes and FEV1/FVC ratios (online supplementary table E6 and figure 2a). Children in Classes 2, 3 and 4 exhibited significantly lower FEV1/FVC ratios than did those in Class $1(\mathrm{p}<0.001)$. Compared with children in Class 1 , those in Class 3 exhibited lower FeNO levels (online supplementary table E6 and figure 2b). Children who experienced a reduction in their BMI z-scores (Class 4) exhibited increased FeNO levels.

\section{TABLE 4 Hazard ratios of asthma/rhinitis phenotypes for the different growth trajectory classes in Cohort 1 aged 12,15 and 18 years}

\begin{tabular}{lccc} 
Trajectory class & Incident asthma & Incident exercise-induced asthma & $\begin{array}{c}\text { Incident allergic rhinitis } \\
\text { I" }\end{array}$ \\
\hline Class 1 (reference) & 1 & 1 & 1 \\
Class 2 & $1.77(0.75-4.15)$ & $0.90(0.60-1.35)$ & $1.14(0.85-1.52)$ \\
Class 3 & $2.47(1.18-5.12)$ & $1.40(1.01-1.95)$ & $1.44(1.12-1.84)$ \\
Class 4 & $1.82(0.38-8.63)$ & $1.68(0.83-3.40)$ & $1.50(0.85-2.64)$
\end{tabular}

Data are presented as hazard ratios with 95\% confidence intervals. See figure 1 and table 2 for details of trajectory classes. \#: discrete time hazard models were adjusted for sex, age, parental education, family income, household environmental tobacco smoke, birthweight, gestational age, breastfeeding, asthma oral steroid use and weighted by individual class probability; ๆ: discrete time hazard models were adjusted for sex, age, parental education, family income, household environmental tobacco smoke, birthweight, gestational age, breastfeeding and weighted by individual class probability. 

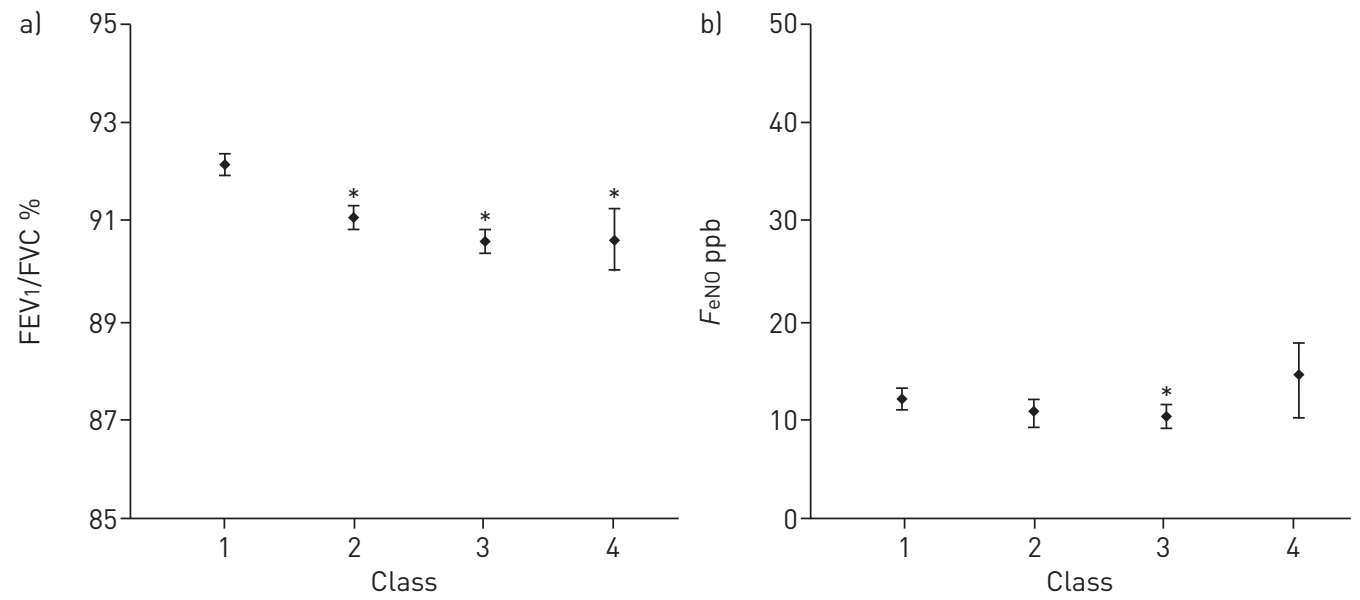

FIGURE 2 a) Forced expiratory volume in $1 \mathrm{~s}$ (FEV 1 )/forced vital capacity (FVC) ratios and b) exhaled nitric oxide fraction $\left(\mathrm{FeNO}_{\mathrm{NO}}\right)$ levels at age 12 years for the four growth trajectory classes (figure 1). *: significant differences $(p<0.05)$ as compared with Class 1 using multivariate ANOVA (MANOVA). MANOVA tests were adjusted for sex, age, parental education, family income, household environmental tobacco smoke, birthweight, gestational age, breastfeeding and asthma oral steroid use.

\section{Discussion}

By using a novel statistical method (i.e. LGMM) we identified four growth pattern classes in Taiwanese children aged 6-11 years: normal growth class, rapid growth class, persistently overweight class and declining obesity class. Persistently overweight children exhibited a significantly increased risk of physician-diagnosed asthma, exercise-induced asthma and allergic rhinitis at age 12 years. Furthermore, our results suggest that the effects of being persistently overweight at age 6-11 years on asthma and rhinitis continued to adolescence and early adulthood. The period of childhood obesity at school age is critical for asthma and rhinitis later in life [30, 31].

Several studies have discovered that rapid growth during infancy significantly predicts asthma in early childhood. One study reported the critical period of infant growth to be between 0 and 2 years [6]. Other studies have indicated that this period is the first 3 months [32, 33]. Our findings that rapid growth in childhood at age 6-11 years did not significantly increase the risk of asthma are consistent with those of previous studies. Moreover, persistent overweight at age 6-11 years may originate from rapid growth in early childhood. Adiposity accumulation at age 6-11 years predicted a long-term effect on incident asthma at age 12, 15 and 18 years (table 4). This implies that the period of being overweight or obese may play different roles in the development of asthma [34]. The children in Class 2 did not exhibit a significantly increased risk of asthma, suggesting that rapid BMI growth in the age range 6-11 years might not be a critical period for asthma development. Weight reduction intervention studies have also reported that weight loss programmes facilitated enhancing symptom control of children with asthma and consequently reducing the use of rescue medications [35].

In the present study, persistently overweight children exhibited a significantly increased risk of allergic rhinitis. This risk can have long-term effects extending into young adulthood. The results of previous studies on the relationships between overweight and allergic rhinitis are not consistent. Certain studies have reported overweight as the risk factor for allergic rhinitis [14], whereas others have reported no associations between overweight and allergic rhinitis [36]. As a comorbidity of asthma, allergic rhinitis is a sequela of atopic diseases incident at the age of approximately 9-17 years. Two studies have reported significant relationships between overweight and allergic rhinitis only in children with wheeze symptoms $[30,37]$. The heterogeneous findings of relevant studies may be due to the different prevalence and incidence rates of allergic rhinitis, and varied participant ages among different countries. Our result is supported by a Taiwanese study which reported that girls with high BMI exhibited an increased risk of rhinitis symptoms [38]. As a result of the high prevalence and incidence rates of allergic rhinitis in Taiwan, and the appropriate age group of participants, our longitudinal study provides an adequate design for examining the relationships between persistent overweight and allergic rhinitis.

Children in Classes 2, 3 and 4 exhibited significantly lower pulmonary function performance levels compared with those in Class 1. A study reported inverse relationships between BMI and FEV1/FVC ratios [39]. Obesity, particularly central obesity [17], might mechanically restrict the diaphragm and limit lung expansion, reflecting impaired lung function. Children in Class 4, despite experiencing a reduction in their 
BMI z-scores, exhibited an extremely higher BMI, which might cause long-term obstruction to the airway, compared with children in Class 1. Previous longitudinal studies discovered that higher weight growth in childhood was associated with higher lung volumes but increased measures of obstruction (FEV1/FVC) [32]. Our findings are consistent with one previous study which reported that in obese individuals, lung function was significantly lower in subjects with greater years of obesity [40]. The underlying mechanism of these associations might include abnormal growth and development of lungs or immunologic or adiposity-related systemic inflammatory effects. Therefore, weight loss programmes should be encouraged to prevent future pulmonary function impairment.

According to our literature review, this is the first study exploring the difference in the airway inflammation status among various growth trajectory classes. Airway inflammation was lower in children with increased BMI z-scores, but it was higher in children with lower BMI z-scores, compared with children in Class I. This finding demonstrating an inverse relationship between obesity and airway inflammation is consistent with the results of previous studies on adults [41] and children [42]. This is because obesity obstructs the airway, affecting the measurement of exhaled nitric oxide. In children demonstrating declined BMI z-scores, the effect of obstruction in the airway by adiposity could be recovered, leading to increased FeNO levels.

The strengths of our study were its nationwide, representative and longitudinal design and follow-up until age 18 years with repeated measurements of BMI and outcomes of asthma and rhinitis. We tested our hypothesis by using two statistical models (logistic regression and DTHM), and the results from these models were consistent, confirming the robustness of our findings. In addition, we conducted pulmonary function tests and collected biomarkers of airway inflammation. A limitation of our study was the use of school health records for collecting body height and weight data of children aged 6-8 years. A validation analysis was conducted by comparing the differences between our measurements and those of the school nurse. We discovered a high correlation ( $\mathrm{r}=0.92, \mathrm{p}<0.001$; data not shown) between these measurements, with only a slight difference occurring between the mean value of these measurements. Moreover, we could not consider the growth factor in early childhood, which might be a crucial period for the development of asthma. However, because the prominent age of onset of allergic rhinitis is approximately 9 years, our study design provides a suitable age group for examining the relationships between different growth patterns and rhinitis. We have also adjusted several perinatal factors, such as birthweight, gestational age and breastfeeding, in the model. Another limitation in this study is that the lack of significance of the risk of asthma in Class 4 is probably due to the relatively small population size $(2.6 \%)$ in this class. In addition, although concurrent BMI might influence the relationships between BMI trajectory class and asthma outcomes, we did not adjust concurrent BMI in the model because it had high collinearity with BMI trajectory class.

In conclusion, persistently overweight children aged 6-11 years exhibited significantly increased risks of asthma and rhinitis, and this effect persisted into adolescence and early adulthood. Measures for preventing obesity should be initiated during school age to reduce the incidence of asthma and rhinitis in later life.

\section{Acknowledgements}

We are grateful to Yu-Kang Tu (Institute of Epidemiology and Preventive Medicine, College of Public Health, National Taiwan University, Taipei, Taiwan) who provided suggestions for the statistics in our research.

Author contributions: Y.-C. Chen contributed the cohort data collection, statistical analysis, interpretation of data and writing. H.-Y. Fan assisted in critical statistical analysis and data management. P.-C. Chen and T.-H. Liou assisted in statistical analysis and data interpretation. B.-L. Chiang and Y.-H. Yang contributed to critically revising this manuscript for intellectual content. Y.L. Lee reviewed the study design and supervised the study.

\section{References}

1 Chen YC, Dong GH, Lin KC, et al. Gender difference of childhood overweight and obesity in predicting the risk of incident asthma: a systematic review and meta-analysis. Obes Rev 2013; 14: 222-231.

2 Beuther DA, Sutherland ER. Overweight, obesity, and incident asthma: a meta-analysis of prospective epidemiologic studies. Am J Respir Crit Care Med 2007; 175: 661-666.

3 Corbo GM, Forastiere F, De Sario M, et al. Wheeze and asthma in children: associations with body mass index, sports, television viewing, and diet. Epidemiology 2008; 19: 747-755.

4 Daniels SR, Arnett DK, Eckel RH, et al. Overweight in children and adolescents: pathophysiology, consequences, prevention, and treatment. Circulation 2005; 111: 1999-2012.

5 Ziyab AH, Karmaus W, Kurukulaaratchy RJ, et al. Developmental trajectories of Body Mass Index from infancy to 18 years of age: prenatal determinants and health consequences. J Epidemiol Community Health 2014; 68: 934-941.

6 Rzehak P, Wijga AH, Keil T, et al. Body mass index trajectory classes and incident asthma in childhood: results from 8 European Birth Cohorts - a Global Allergy and Asthma European Network initiative. J Allergy Clin Immunol 2013; 131: 1528-1536.

7 Anderson HR, Pottier AC, Strachan DP. Asthma from birth to age 23: incidence and relation to prior and concurrent atopic disease. Thorax 1992; 47: 537-542. 
8 Kellberger J, Dressel H, Vogelberg C, et al. Prediction of the incidence and persistence of allergic rhinitis in adolescence: a prospective cohort study. J Allergy Clin Immunol 2012; 129: 397-402.

9 de Bot CMA, Moed H, Schellevis FG, et al. Allergic rhinitis in children: incidence and treatment in Dutch general practice in 1987 and 2001. Pediatr Allergy Immunol 2009; 20: 571-577.

10 Juniper EF, Ståhl E, Doty RL, et al. Clinical outcomes and adverse effect monitoring in allergic rhinitis. J Allergy Clin Immunol 2005; 115: S390-S413.

11 Sybilski AJ, Raciborski F, Lipiec A, et al. Obesity - a risk factor for asthma, but not for atopic dermatitis, allergic rhinitis and sensitization. Public Health Nutr 2015; 18: 530-536.

12 Kreißl S, Radon K, Dressel H, et al. Body mass index change and atopic diseases are not always associated in children and adolescents. Ann Allergy Asthma Immunol 2014; 113: 440-444.

13 Bråbäck L, Hjern A, Rasmussen F. Body mass index, asthma and allergic rhinoconjunctivitis in Swedish conscripts - a national cohort study over three decades. Respir Med 2005; 99: 1010-1014.

14 Baumann LM, Romero KM, Robinson CL, et al. Prevalence and risk factors for allergic rhinitis in two resource-limited settings in Peru with disparate degrees of urbanization. Clin Exp Allergy 2015; 45: $192-199$.

15 Chen YC, Chen PC, Hsieh WS, et al. Environmental factors associated with overweight and obesity in Taiwanese children. Paediatr Perinat Epidemiol 2012; 26: 561-571.

16 World Medical Association Declaration of Helsinki. Recommendations guiding physicians in biomedical research involving human subjects. JAMA 1997; 277: 925-926.

17 Chen YC, Tu YK, Huang KC, et al. Pathway from central obesity to childhood asthma. Physical fitness and sedentary time are leading factors. Am J Respir Crit Care Med 2014; 189: 1194-1203.

18 de Onis M, Onyango AW, Borghi E, et al. Development of a WHO growth reference for school-aged children and adolescents. Bull World Health Organ 2007; 85: 660-667.

19 WHO Multicenter Growth Reference Study Group. WHO child growth standards: length/height-for-age, weight-for-age, weight-for-length, weight-for height and body mass index-for-age: methods and development. www.who.int/childgrowth/standards/second_set/technical_report_2/en/Date last accessed: October 4, 2016.

20 Chen W, Chang MH. New growth charts for Taiwanese children and adolescents based on World Health Organization standards and health-related physical fitness. Pediatr Neonatol 2010; 51: 69-79.

21 Cole TJ, Lobstein T. Extended international (IOTF) body mass index cut-offs for thinness, overweight and obesity. Pediatr Obes 2012; 7: 284-294.

22 Lee YL, Chen YC, Chen YA. Obesity and the occurrence of bronchitic symptoms in adolescents. Obesity (Silver Spring) 2012; 21: E149-E535.

23 Dweik RA, Boggs PB, Erzurum SC, et al. An official ATS clinical practice guideline: interpretation of exhaled nitric oxide levels ( $\mathrm{FE}_{\mathrm{NO}}$ ) for clinical applications. Am J Respir Crit Care Med 2011; 184: 602-615.

24 American Thoracic Society, European Respiratory Society. ATS/ERS recommendations for standardized procedures for the online and offline measurement of exhaled lower respiratory nitric oxide and nasal nitric oxide, 2005. Am J Respir Crit Care Med 2005; 171: 912-930.

25 Lee YL, Hwang BF, Chen YA, et al. Pulmonary function and incident bronchitis and asthma in children: a community-based prospective cohort study. PLoS One 2012; 7: e32477.

26 Jung T, Wickrama KAS. An introduction to latent class growth analysis and growth mixture modeling. Soc Personal Psychol Compass 2008; 2: 302-317.

27 Muthen B, Brown $\mathrm{CH}$, Masyn $\mathrm{K}$, et al. General growth mixture modeling for randomized preventive interventions Biostatistics 2002; 3: 459-475.

28 Tein JY, Coxe S, Cham H. Statistical power to detect the correct number of classes in latent profile analysis. Struct Equ Modeling 2013; 20: 640-657.

29 Allison PD, ed. Survival Analysis Using SAS: A Practical Guide. Cary, SAS Institute, 2010.

30 Saadeh D, Salameh P, Caillaud D, et al. High body mass index and allergies in schoolchildren: the French six cities study. BMJ Open Respir Res 2014; 1: e000054-e000054.

31 Mannino DM, Mott J, Ferdinands JM, et al. Boys with high body masses have an increased risk of developing asthma: findings from the National Longitudinal Survey of Youth (NLSY). Int J Obesity (Lond) 2006; 30 : 6-13.

32 Sonnenschein-van der Voort AMM, Howe LD, Granell R, et al. Influence of childhood growth on asthma and lung function in adolescence. J Allergy Clin Immunol 2015; 135: 1435-1443.

33 van der Gugten AC, Koopman M, Evelein AMV, et al. Rapid early weight gain is associated with wheeze and reduced lung function in childhood. Eur Respir J 2012; 39: 403-410.

34 Scholtens S, Wijga AH, Seidell JC, et al. Overweight and changes in weight status during childhood in relation to asthma symptoms at 8 years of age. J Allergy Clin Immunol 2009; 123: 1312-1318.

35 Adeniyi FB, Young T. Weight loss interventions for chronic asthma. Cochrane Database Syst Rev 2012; 7: CD009339.

36 Sidell D, Shapiro NL, Bhattacharyya N. Obesity and the risk of chronic rhinosinusitis, allergic rhinitis, and acute otitis media in school-age children. Laryngoscope 2013; 123: 2360-2363.

37 Weinmayr G, Forastiere F, Büchele G, et al. Overweight/obesity and respiratory and allergic disease in children: international study of asthma and allergies in childhood (ISAAC) phase two. PLoS One 2014; 9: e113996-e113996.

38 Huang SL, Shiao G, Chou P. Association between body mass index and allergy in teenage girls in Taiwan Clin Exp Allergy 1999; 29: 323-329.

39 Davidson WJ, Mackenzie-Rife KA, Witmans MB, et al. Obesity negatively impacts lung function in children and adolescents. Pediatr Pulmonol 2014; 49: 1003-1010.

40 Santamaria F, Montella S, Greco L, et al. Obesity duration is associated to pulmonary function impairment in obese subjects. Obesity (Silver Spring) 2011; 19: 1623-1628.

41 McLachlan CR, Poulton R, Car G, et al. Adiposity, asthma, and airway inflammation. J Allergy Clin Immunol 2007; 119: 634-639.

42 Santamaria F, Montella S, De Stefano S, et al. Asthma, atopy, and airway inflammation in obese children. J Allergy Clin Immunol 2007; 120: 965-967. 